\title{
Augenöffner
}

\section{Anna Sax}

Lic. oec. publ., MHA, Mitglied der Redaktion

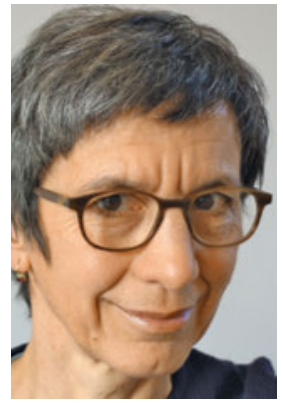

Kürzlich ist mir ein Buch untergekommen, das mich in Erstaunen versetzt und auch empört hat: Manche Leute werden für dement gehalten und psychiatrisiert, und das nur, weil sie nicht gut sehen und es niemand bemerkt hat. Und wenn doch klar wird, dass es sich um eine Sehbehinderung handelt, gibt es keine Regelung für Integrationsmassnahmen. Weil die Betroffenen alt sind. Wozu und in was sollen sehbehinderte und pflegebedürftige alte Menschen denn noch integriert werden? Sie sollen froh sein, wenn Sie ein warmes Bett und zu essen haben, wenn jemand da ist, der sie pflegt und jemand, der ihnen ab und zu etwas vorliest. Eine alte Frau, ein alter Mann hat keine Aufgaben mehr zu erfüllen und somit keine Funktion in unserer Gesellschaft. Wer im hohen Alter schlecht sieht oder schlecht hört, hat sich damit abzufinden. Viele Leute, auch Betroffene, sehen das leider so.

Obwohl selbst Laien wissen, dass die Sehkraft mit dem Älterwerden meistens nachlässt, erkennen oftmals weder das Umfeld noch die Betroffenen selbst eine Sehbehinderung. Das hat auch damit zu tun, dass wir «mit dem Gehirn sehen». Dieses kann zum Beispiel ein Bild vervollständigen, wenn ein Teil des Blickfeldes ausfällt («Filling-in») oder visuelle Halluzinationen produzieren ("Charles-Bonnet-Syndrom»). Die Folge ist, dass jemand, dessen Gesichtsfeld in der Mitte blind ist, zwar den Tisch sieht, nicht aber den Teller, der darauf steht. Oder dass jemand Hindernisse sieht, wo keine sind. Und nun? Was halten wir von einem Grossvater, der sich darüber beschwert, dass alle um ihn herum Wein trinken und er als einziger kein Glas bekommen hat, obwohl es vor ihm steht? Oder von der Schwiegermutter, die ständig fürchtet, in nicht vorhandene Löcher zu treten? Ist doch logisch: Sie sind dement. Angehörige denken das, und früher oder später glauben es die Betroffenen selbst. Doch es kommt noch schlimmer: Auch Fachleute halten sehbehinderte alte Menschen oft für dement, wie eine im Buch vorgestellte Untersuchung zu Katarakt-Patientinnen und Patienten zeigt. Der Verlust der Sehkraft scheint nämlich unerkannt und unbehandelt zu kognitiven Verlusten zu führen, die einer Demenz täuschend ähnlich sehen. Doch es zeigt sich, dass ursprünglich als dement eingeschätzte Personen nach einer Katarakt- operation ihre kognitiven Fähigkeiten wiedererlangen. Eine weitere Studie kommt zum Schluss, dass viele Bewohnerinnen von Pflegeinstitutionen mit einer Sehbehinderung eine Pflege erhalten, die auf Demenz fokussiert. Mit einer rehabilitativ ausgerichteten Unterstützung zur Selbstständigkeit könnte die wahre Häufigkeit von Demenz deutlich reduziert werden, folgern die Buch-Herausgeberinnen. Auch Depression ist häufig Folge einer Sehbehinderung und könnte mit entsprechender Begleitung und Pflege vermieden werden. Es muss wohl kaum erwähnt werden, dass bei Verlusten der Hörfähigkeit im Alter ähnliche Phänomene zu beobachten sind.

Das Buch ist im wahrsten Sinne des Wortes ein Augenöffner. Auch wer im Alter die Fähigkeit zu sehen und zu hören verliert, kann mit befähigender Unterstützung selbstbestimmt leben und aktiv am Leben teilnehmen. Die Herausgeberinnen und die Autorinnen zeigen anhand vieler Beispiele aus ihrer langen Praxis, wie es gehen könnte. Die Essenz des Buches bildet ein Leitfaden mit 33 goldenen Regeln, wie einer Sehbehinderung im Alter angemessen zu begegnen ist. Die Geschichte hat auch eine politische Dimension: Das Sozialversicherungssystem sieht keine angemessene Unterstützung für Menschen vor, die im Alter eine Behinderung erwerben. Die Zeit der Berufstätigkeit ist vorbei, damit ist die IV nicht mehr zuständig für die Eingliederung. Im Alter ist die Krankenversicherung zuständig. Diese bezahlt für die Pflege und die medizinische Versorgung, aber nicht für eine aktive Lebensgestaltung. Es wäre an der Zeit, die soziale Integration gerade im Alter als politische Aufgabe ernst zu nehmen und entsprechende Massnahmen zu unterstützen, beispielsweise über Ergänzungsleistungen zur AHV. Damit helfen wir nicht nur den Betroffenen, sondern entlasten auch das Gesundheits- und Sozialsystem. Denn je länger wir im Alter selbstbestimmt leben, desto eher können wir auch etwas für unsere Mitmenschen tun.

\section{Literatur}

Fatima Heussler, Judith Wildi und Magdalena Seibl (Hrsg.), Menschen mit Sehbehinderung in Alterseinrichtungen. Gerontagogik und gerontagogische Pflege - Empfehlungen zur Inklusion, Seismo Verlag, Zürich 2016 